\title{
Decreases in Elective and Non-Elective Surgical Case Volumes During the COVID-19 Pandemic
}

\author{
Sukriti Bansal ${ }^{\mathrm{a}, \mathrm{c}}$, Youmna A. Sherif ${ }^{\mathrm{a}, \mathrm{c}, \mathrm{d}}$, Rachel W. Davis ${ }^{\mathrm{a}}$, Marcia Barnett ${ }^{\mathrm{a}}$, Umang M. Parikh ${ }^{\mathrm{a}}$, \\ Hunter Bechtold ${ }^{\mathrm{a}}$, Hudson Holmes ${ }^{\mathrm{a}}$, Yao Yang ${ }^{\mathrm{a}}$, David Holmes ${ }^{\mathrm{a}}$, Megan Vu ${ }^{\mathrm{a}}$, \\ Jed G. Nuchtern ${ }^{\mathrm{a}, \mathrm{b}}$, Chad T. Wilson ${ }^{\mathrm{a}}$
}

\begin{abstract}
Background: The coronavirus disease 2019 (COVID-19) pandemic has had an unprecedented impact on surgical healthcare delivery systems. Multiple surgical organizations outlined recommendations on the performance of surgeries to minimize viral transmission, prioritize resource allocation, and avoid perioperative complications. This study aims to characterize the changes in surgical volume during the COVID-19 pandemic.

Methods: A retrospective chart review was performed at a large public hospital to characterize the surgical case volume, specialties performing surgeries, case urgency (elective vs. non-elective), patient presentation (emergency room, clinic, inpatient), and patient demographics. Data were collected between January 17 and May 8, 2020, 8 weeks prior to and 8 weeks after the declaration of COVID-19 as a national emergency in the USA. For comparison, data between January 17 and May 8, 2019 were also collected. A univariate analysis was performed via paired tests between the two years.
\end{abstract}

Results: There was a statistically significant decrease in both elective and non-elective cases in 2020. When compared to 2019, the weekly case volume in 2020 is significantly higher prior to the declaration of COVID-19 a national emergency (weeks 1 - 8) and significantly lower after the declaration of COVID-19 as a national emergency (weeks 9 - 16). Additionally, there appeared to be statistically significant decrease in non-elective surgical case volumes.

Conclusions: In facing the challenges presented by the COVID-19 pandemic, clinician leaders have been tasked with making difficult decisions regarding patient care. While leading surgical organizations provided guidelines for best practices at the start of the pandemic, the

Manuscript submitted November 21, 2021, accepted December 13, 2021

Published online December 31, 2021

${ }^{a}$ Michael E. DeBakey Department of Surgery, Baylor College of Medicine, Houston, TX 77030, USA

'Texas Children's Hospital, Houston, TX 77030, USA

'These authors contributed equally to this article.

${ }^{\mathrm{d} C}$ Corresponding Author: Youmna A. Sherif, Michael E. DeBakey Department of Surgery, Baylor College of Medicine, Houston, TX 77030, USA.

Email: sherif@bcm.edu

doi: https://doi.org/10.14740/jcs452 long-term implications of these decisions are unknown. This study has found that the COVD-19 pandemic has resulted in decreased volume of both elective and non-elective surgeries, raising concerns that necessary care may be delayed for marginalized populations.

Keywords: COVID-19; Surgical case volumes; Resource-limited; Delay in care

\section{Introduction}

The coronavirus disease 2019 (COVID-19) pandemic has had an unprecedented impact on the American healthcare delivery system, healthcare infrastructure, and clinical workforce. In an effort to limit the spread of the virus, hospital systems have employed various measures such as encouraging the use of masks and face shields [1], increasing the use of telemedicine [2], and designating specific COVID-19 units [3]. Similarly, surgical organizations outlined specific recommendations for the management of operative pathologies during the pandemic. These recommendations emphasize the need to: 1 ) decrease the transmission of COVID-19 during the preoperative, intraoperative, and postoperative period [4];2) limit perioperative complications related to COVID-19 [5]; and 3) ensure appropriate resource allocation and conservation.

The American College of Surgeons (ACS) clarified the need for a brief cessation of elective cases during the pandemic's initial peaks and a subsequent, thoughtful reintroduction of elective cases in local facilities [6]. Additionally, the ACS encouraged the conservative medical management of certain diseases when appropriate in COVID-positive patients, adoption of a triaging system for non-emergent operative cases, and provision of appropriate personal protective equipment when operating on COVID-positive patients.

In following these guidelines, the nature of surgery and anesthesia practices changed during the COVID-19 pandemic as most elective and non-urgent cases were postponed. This decision raised multiple concerns within the oncologic community as delay by even 1 month can increase mortality in patients with cancer [7]. Additionally, pathology requiring urgent and emergent interventions during the pandemic differed from prior periods. For example, there was a noted increase in gunshot wounds [8], a decrease in blunt trauma (often attrib- 
uted to restrictions on movement), and an overall decrease in operative trauma [9].

The uncertainty that COVID-19 created and the delays in the delivery of surgical care it imposed forced the surgeon into a different role. Surgeons were tasked with identifying patients who can be safely diverted to non-operative management and prioritizing the disposition of patients with an urgent need for surgical intervention. To understand the impact of COVID-19, it is important to assess the changes in surgical case volumes, and more importantly the changes in non-elective case volumes, during the pandemic. This is critical in order to clarify the need for patient follow-up and important considerations regarding the progression of disease beyond optimal surgical management.

\section{Materials and Methods}

\section{Data collection}

This study is a retrospective chart review that compares surgical case volumes at a large public hospital during a 16-week period (January 17 to May 8) in 2019 to the same 16-week period in 2020. This 16-week period was chosen as it spans both the declaration of the COVID-19 pandemic as a national emergency in the USA (March 13, 2020) and the period in which all elective cases were cancelled by the governor of the state of Texas (March 22 to April 21). Data were collected for the same dates in 2019 to account for seasonal variability.

The electronic medical records (EMRs) of all patients who underwent surgery during this time were reviewed, and data were collected in a deidentified fashion. Data included surgeries performed, specialties performing the surgeries, case classification, patient presentation, patient demographics, and COVID status if available. Data were stored in an encrypted database. A total of 12 surgical specialties were represented in the study including acute care and trauma surgery, cardiothoracic surgery, endocrine surgery, ophthalmology, oral and maxillofacial surgery, neurosurgery, otolaryngology, orthopedic surgery, plastic surgery, surgical oncology, urology, and vascular surgery. Case classification included four categories as defined by the hospital: elective (defined as no time limit to case start from posting), urgent (case must start within 6 $\mathrm{h}$ of posting), emergent (case required to start within $3 \mathrm{~h}$ of posting), and STAT (case to start upon immediate arrival of patient). Case classification was dichotomized into elective and non-elective, where non-elective cases included urgent, emergent, and STAT cases. Patient presentation was comprised of three sources: clinic, emergency room (ER), and inpatient. In terms of patient demographics, information regarding age, race, ethnicity, and insurance status was collected.

For this study, the obstetrics and gynecology data were not included as the service has a dedicated operating suite with separate operative case logs and restricted access.

\section{Data analysis}

A non-parametric paired test was performed to compare the weekly case volumes by classification, specialty, and presentation by pairing the weeks in 2019 and 2020 comparing them. These data were then grouped in two cohorts: 1) cases in weeks 1 - 8 (corresponding dates Jan 17 to March 13); 2) cases in weeks 9 - 16 (dates March 14 to May 8), to represent the dates before and after the declaration of COVID-19 as a national emergency. The paired results for the two cohorts were analyzed using a signed rank sum test for each objective $(\alpha \leq 0.05)$. The change in surgical case volumes for total and non-elective surgical cases from 2019 to 2020 before and after COVID was assessed. Secondary objectives included evaluating the change in total case volumes and non-elective case volumes for each specialty in 2019 and 2020 between Jan 17 and May 8.

\section{Ethical approval}

This study was reviewed by the Baylor College of Medicine Institutional Review Board and the Harris County Health System Institutional Review Board, and was determined to be an exempt study by both; and the study was conducted in compliance with the ethical standards of the Baylor College of Medicine and Harris Health Institutional Review Boards.

\section{Results}

\section{Population}

Overall, the cohort was 53\% female for both years (Table 1). The mean age was $48.2 \pm 15.2$ years in 2019 and $46.8 \pm 15$ years in $2020(\mathrm{P}=0.0012)$ (Table 2$)$. During 2019, the patient population identified as 46\% Hispanic, 25\% African American, $16 \%$ Caucasian, 2.6\% Asian, and 10\% other, with other races compromising $<1 \%$ of the cohort (Table 1 , race as defined in EMR). In the 2020 cohort, patients self-identified as 39\% Hispanic, 27\% African American, 17\% Caucasian, 2\% Asian, and $14 \%$ other, with other races compromising $<1 \%$ of the population (Table 1). This represents a difference in the racial demographics of the population between the two years.

For the 2019 cohort of patients, insurance sources were as follows: $21 \%$ self-pay, $49 \%$ county-subsidized insurance, $7.5 \%$ Medicaid, $8.3 \%$ Medicare, and 14\% private insurance (Table 1). In 2020, the patient insurance sources were as follows: $18 \%$ self-pay, $54 \%$ county subsidized insurance, $7.7 \%$ Medicaid, 7.7\% Medicare, and 13\% private insurance (Table $1)$. This represents a statistically significant difference in distribution of payor source $(\mathrm{P}=0.0007)$ between the two years.

\section{Patient presentation}

Of the 2,745 patients who underwent surgery in 2019, 535 (19.5\%) presented from the ER, 794 (28.9\%) presented from the inpatient setting, and the remaining 1,416 (51.6\%) presented from clinic. During 2020, a total of 2,311 patients underwent surgery, of whom two did not have presentation data. Of 
Table 1. Summary Demographic Information Between 2019 and 2020

\begin{tabular}{|c|c|c|}
\hline Variable name and indicators & 2019, n (\%) & 2020, n (\%) \\
\hline \multicolumn{3}{|l|}{ Sex } \\
\hline Male & $1,277(47)$ & $1,096(47)$ \\
\hline \multicolumn{3}{|l|}{ Race } \\
\hline African American & $684(25)$ & $622(27)$ \\
\hline Asian & $70(2.6)$ & $46(2)$ \\
\hline Caucasian & $438(16)$ & $385(17)$ \\
\hline Native American/Alaska Native & $3(0.11)$ & $3(0.13)$ \\
\hline Native Hawaiian and Pacific Island & $4(0.15)$ & $0(0)$ \\
\hline Other & $280(10)$ & $330(14)$ \\
\hline County subsidized & $1,337(49)$ & $1,255(54)$ \\
\hline Medicaid & $205(7.5)$ & $179(7.7)$ \\
\hline Medicare & $228(8.3)$ & $179(7.7)$ \\
\hline Private & $388(14)$ & $289(13)$ \\
\hline
\end{tabular}

the remaining 2,309 patients undergoing surgery, $530(23 \%)$ presented through the ER, $653(28.3 \%)$ presented as inpatient, and $1,126(48.8 \%)$ presented from the clinic setting. This represented a statistically significant change in the distribution of patient presentation for surgery $(\mathrm{P}=0.0095)$. During the first 8 weeks of 2020, the distribution of patients presenting from each setting was $274(17.5 \%)$ from the ER, $428(27.3 \%)$ as inpatient, and $864(55.2 \%)$ from clinic. The second 8 weeks of 2020 saw $256(34.5 \%)$ patients present from the ER, 225 $(30.3 \%)$ patients presenting as inpatient, and $262(35.3 \%)$ patients present through clinic (Fig. 1).

\section{Overall surgical volume}

A total of 2,745 surgeries were performed from January 17 to May 8, 2019 and a total of 2,311 surgeries were performed during the same period in 2020. The weekly case volume in 2019 was stable across all 8 weeks (mean $=172 \pm 11$ ) (Fig. 2). Conversely, the mean weekly case volume in 2020 was $192 \pm 23$ prior to March 13 and decreased to $93 \pm 24$ after March 13. This was a statistically significant decrease in case volumes from the first 8 weeks and the second 8 weeks $(\mathrm{P}=0.007)$ (Fig. 3). When compared to 2019, the decrease in surgical case volumes between

Table 2. Average Age of Participants in 2019 and 2020

\begin{tabular}{lll}
\hline Variable & $\mathbf{2 0 1 9}$ & $\mathbf{2 0 2 0}$ \\
\hline Age $($ mean \pm SD) & $48.2 \pm 15.2$ & $46.8 \pm 15$ \\
\hline
\end{tabular}

SD: standard deviation.
March 13 and May 8 of 2020 remained statistically significant.

\section{Non-elective surgical case volumes}

There were 1,535 and 1,250 non-elective surgeries performed in 2019 and 2020, respectively (Fig. 4). Prior to the announcement of COVID-19 as a national emergency in 2020 the mean weekly non-elective surgical volume was $87 \pm 9$ (weeks 1 $8)$. After the announcement of COVID-19 as a national emergency, the mean weekly non-elective surgical volume was 70 \pm 17 (weeks 9 - 16). The weekly case volume was consistent throughout 2019 (mean $=96 \pm 10$ ) and significantly higher volume in the last 8 weeks of 2019 when compared to 2020 $(\mathrm{P}=0.02)$.

\section{Surgical case volume by specialty}

A total of 12 surgical specialties are represented at this large public institution. Case volumes varied by specialty.

\section{Acute care and trauma surgery}

In 2019, the acute care and trauma surgery service performed a total of 598 cases between January 17 and May 8, of which 467 cases were non-elective. During the same period in 2020, a total of 475 cases were performed, including 372 non-elective cases. Prior to the declaration of COVID-19 as a national 


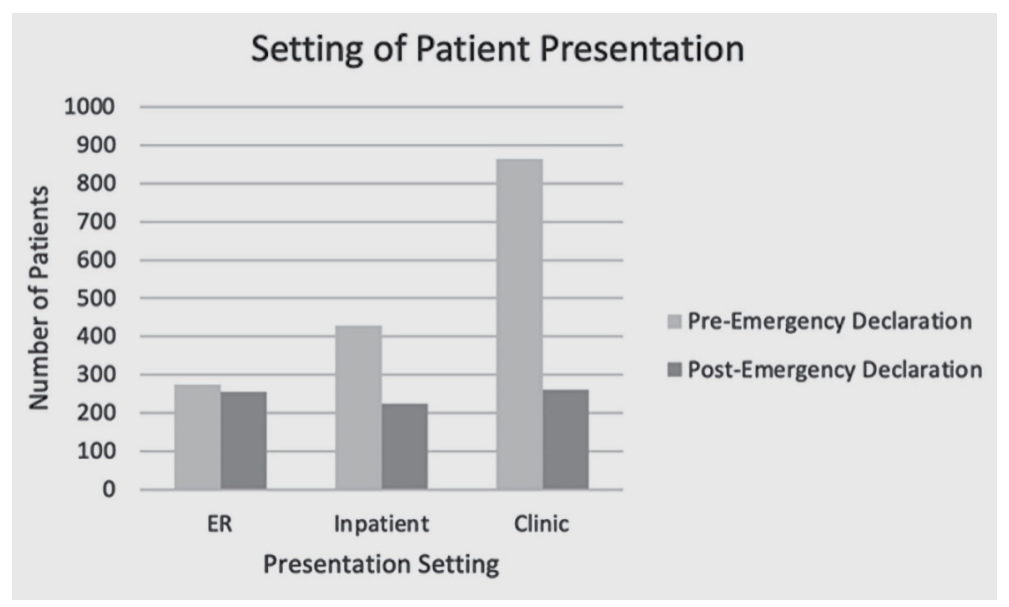

Figure 1. The setting in which patients presented for surgical care between March 14 and May 8 in 2019 and 2020. ER: emergency room.

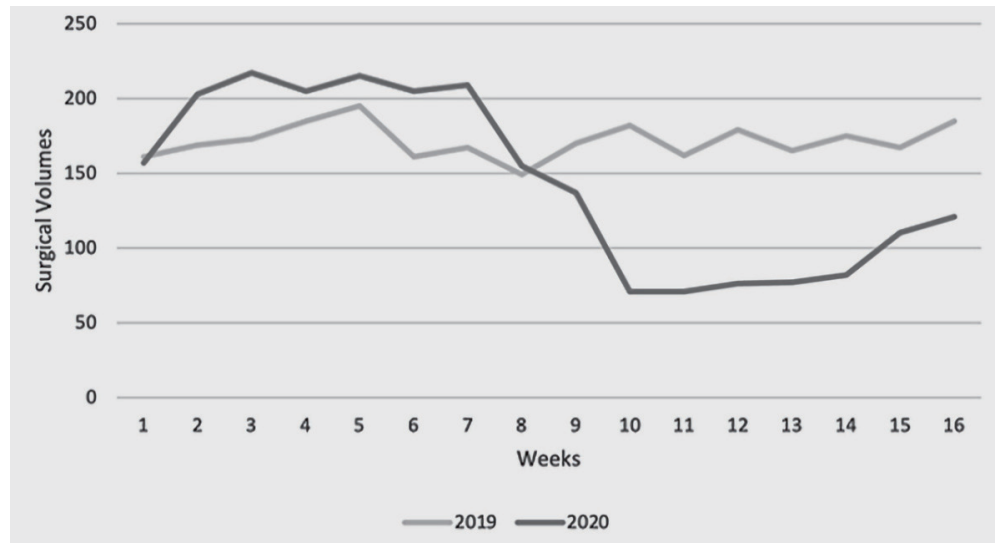

Figure 2. Total number of surgical cases by week between January 17 and May 8 in 2019 and 2020.

emergency (weeks 1 - 8), there was no significant difference in total weekly case volumes compared to the prior year $(\mathrm{P}$ $=0.414)$ (Fig. 5a), nor was there a significant difference in non-elective weekly case volumes $(\mathrm{P}=0.9844)$ (Fig. 6a). After the declaration, a statistically significant decrease in both total case volume ( $\mathrm{P}=0.0078)$ (Fig. 5a), and non-elective case vol- ume $(\mathrm{P}=0.0469)($ Fig. 6a) was noted.

Cardiothoracic surgery

The cardiothoracic surgery service performed 75 total cases

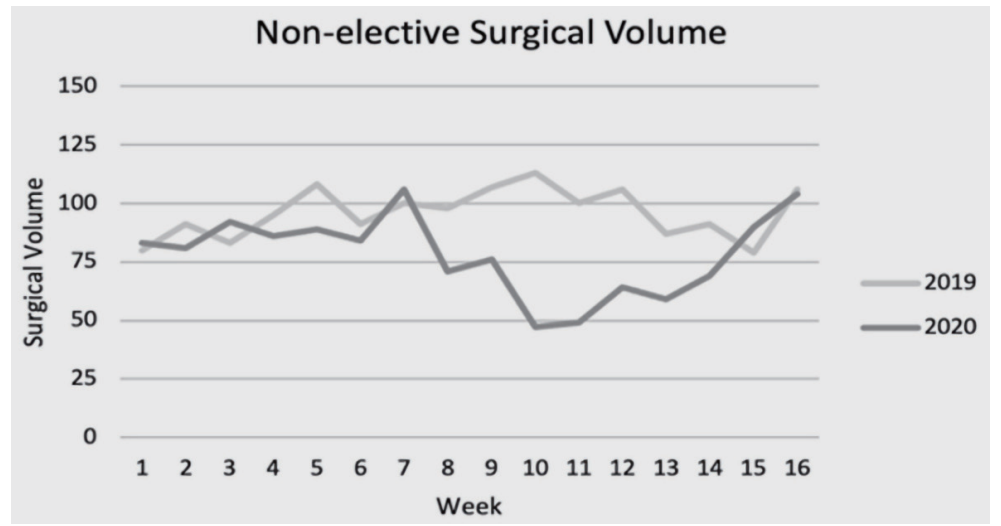

Figure 3. Weekly non-elective surgical case volume between January 17 and May 8 in 2019 and 2020. 


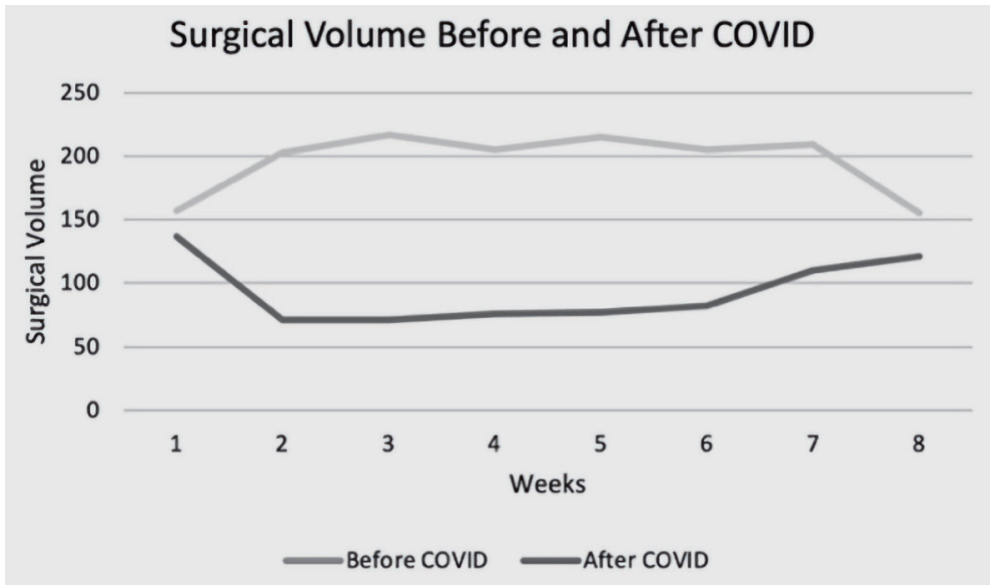

Figure 4. Total weekly surgical volume before and after the declaration of COVID-19 a national emergency. COVID-19: coronavirus disease 2019.
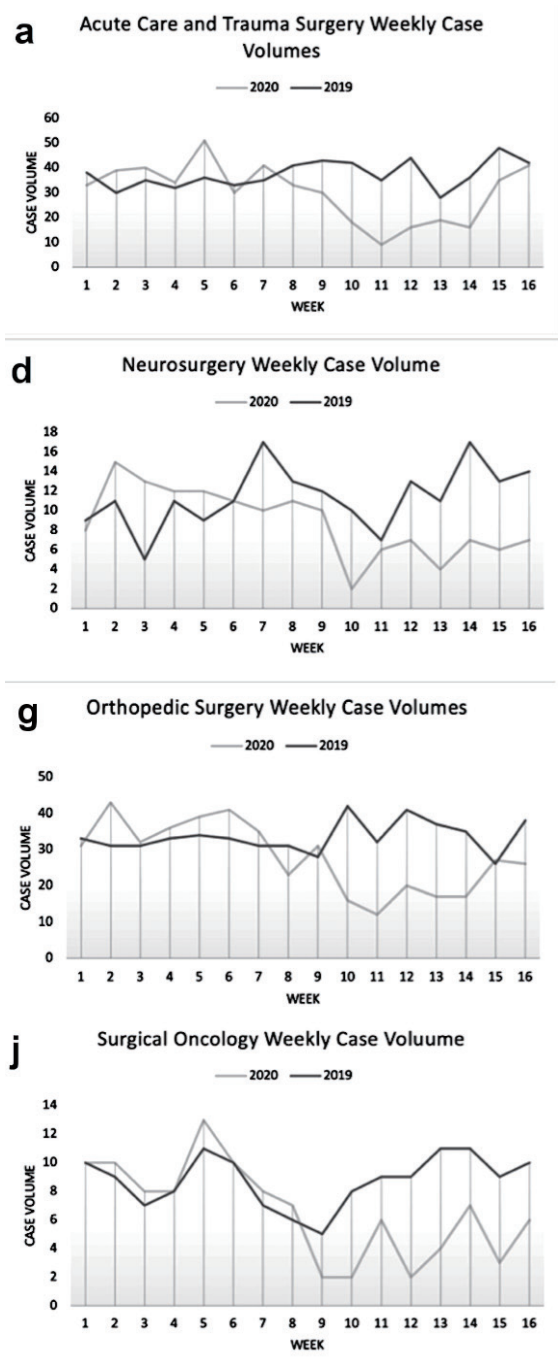
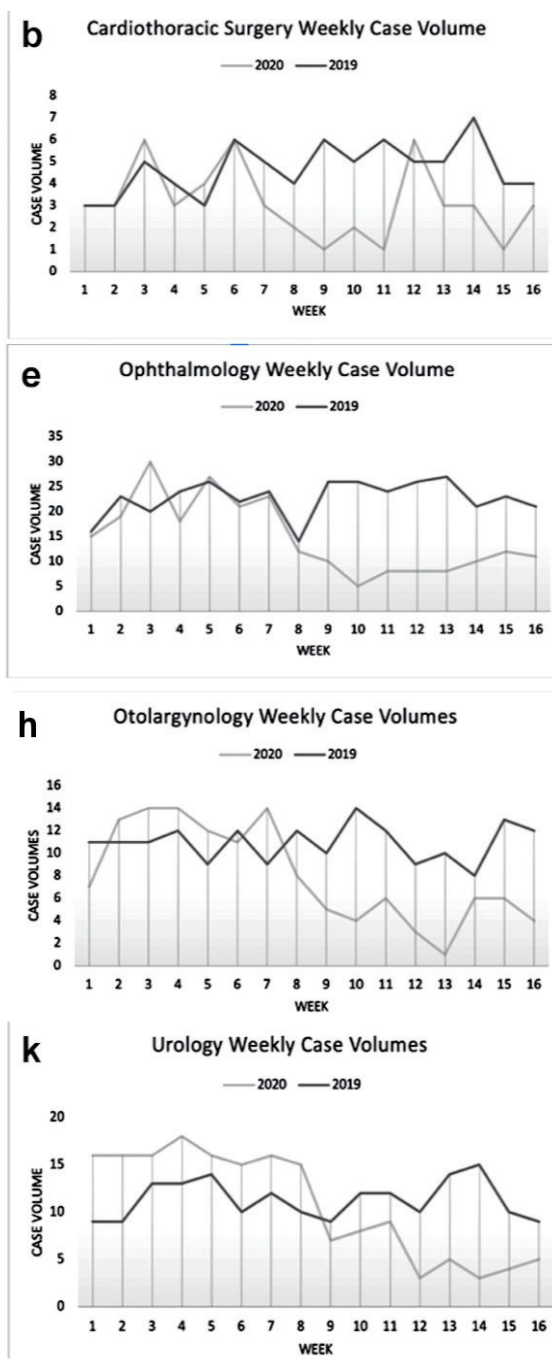
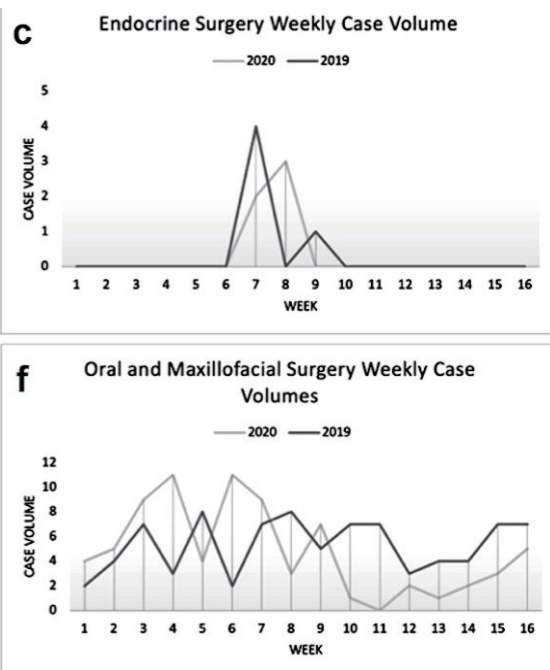
I Plastic and Reconstructive Surgery Weekly Case
Volumes

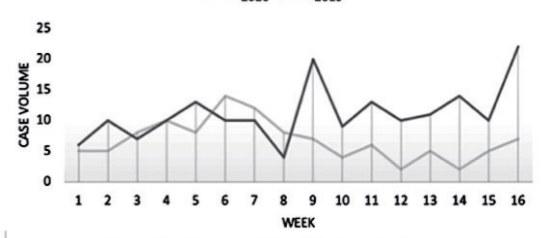

| Vascular Surgery Weekly Case Volumes

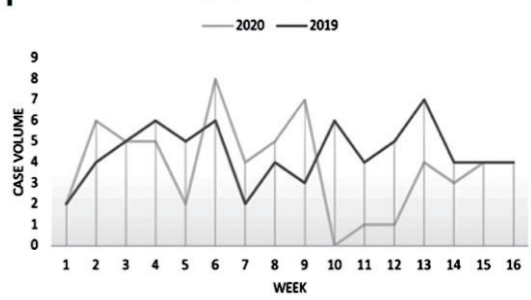

Figure 5. Surgical case volumes by specialty between January 17 and May 8 in 2019 and 2020. (a) Acute care and trauma surgery. (b) Cardiothoracic surgery. (c) Endocrine surgery. (d) Neurosurgery. (e) Ophthalmology. (f) Oral and maxillofacial surgery. (g) Orthopedic surgery. (h) Otolaryngology. (i) Plastic surgery. (j) Surgical oncology. (k) Urology. (l) Vascular surgery. 

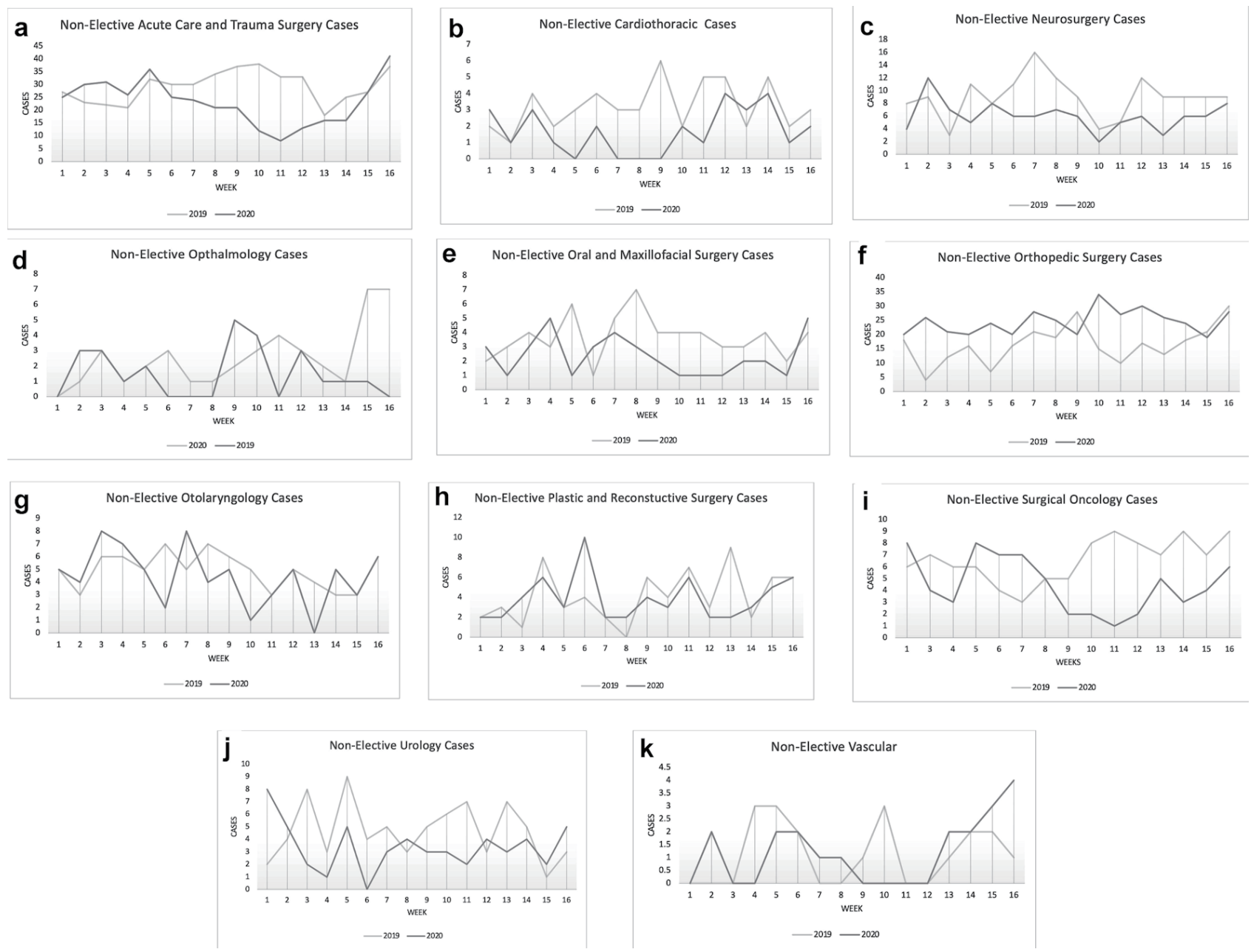

Figure 6. Non-elective case volumes between January 17 and May 8 in 2019 and 2020 categorized by specialty. (a) Acute care and trauma surgery. (b) Cardiothoracic surgery. (c) Neurosurgery. (d) Ophthalmology. (e) Oral and maxillofacial surgery. (f) Orthopedic surgery. (g) Otolaryngology. (h) Plastic surgery. (i) Surgical oncology. (j) Urology. (k) Vascular surgery.

and 52 non-elective cases from January 17 to May 8, 2019. In 2020 , over the same period, 50 total cases and 27 non-elective cases were performed. Prior to the declaration of COVID-19 as a national emergency (weeks $1-8$ ), there was no significant difference in weekly total case volumes $(\mathrm{P}=0.437)$ (Fig. $5 b)$ or weekly non-elective case volumes $(\mathrm{P}=0.0625)$ (Fig. 6b) compared to 2019 . There was a statistically significant drop in total case volume $(\mathrm{P}=0.0234)$ after the declaration of COVID-19 as a national emergency (weeks 9 - 16) (Fig. $5 b)$. The difference in weekly non-elective case volumes after the declaration was not statistically significant $(P=0.0938)$ (Fig. 6b)

\section{Endocrine surgery}

The endocrine surgery service performed five total cases between January 17 to May 8 in both 2019 and 2020 (Fig. 5c). Of those cases three were non-elective in 2019 and two were non-elective in 2020.

\section{Neurosurgery}

In 2019 , the neurosurgery service performed 183 cases from January 17 to May 8 and 141 total cases in 2020. There were 144 non-elective cases in 2019 during that period and 97 in 2020. During weeks $1-8$, there was no significant difference in total weekly case volumes compared to $2019(\mathrm{P}=0.609)$ (Fig. 5d).There was a statistically significant decrease in total case volume ( $\mathrm{P}=0.0234)$ during weeks 9 - 16 (Fig. 5d). The difference in non-elective weekly case volume was not statistically significant for weeks $1-8$ but was significant for weeks 9 - $16(\mathrm{P}=0.0156)$ (Fig. 6c).

\section{Ophthalmology}

A total of 363 cases were performed by the ophthalmology service from January 17 to May 8 in 2019 and 237 total cases were performed in 2020. Twenty-four non-elective cases were performed by the service in 2019, and 41 non-elective cases 
in 2020. There was no significant difference in total weekly case volumes between 2019 and 2020 during the January 17 to March 13 period $(\mathrm{P}=0.328)$ (Fig. 5e), nor was there a significant difference in non-elective cases over that same period $(\mathrm{P}=$ 0.625 ) (Fig. 6d). There was a statistically significant decrease in total case volumes $(\mathrm{P}=0.0078)$ (Fig. 5e), but not in nonelective case volume $(\mathrm{P}=0.25)$ during the March 14 to May 8 period (Fig. 6d).

\section{Oral and maxillofacial surgery}

The oral and maxillofacial surgery service performed 85 total cases and 59 non-elective cases from January 17 to May 8, 2019 and 77 total cases and 38 non-elective cases in 2020 . Again, a statistically significant decrease in total cases was noted in weeks 9 - 16 in $2020(P=0.0391)$ (Fig. 5f), and nonelective cases $(\mathrm{P}=0.0313)$ (Fig. 6e).

\section{Orthopedic surgery}

For orthopedic surgery, 536 total cases were performed between January 17 and May 8 in 2019, and 446 total cases were performed in 2020. A total of 392 non-elective cases were performed in 2019, and 265 non-elective cases in 2020. A statistically significant drop in total case volume between 2019 and 2020 was noted in weeks $9-16(\mathrm{P}=0.0391)$ (Fig. 5g). There was a significant increase in non-elective case volume for weeks $1-8$ between 2019 and $2020(\mathrm{P}=0.0078)$ (Fig. 6f), and no difference in non-elective case volume for weeks 9 - 16 between 2019 and $2020(\mathrm{P}=0.1563)$.

\section{Otolaryngology}

In 2019, the otolaryngology service performed 175 total cases and 79 non-elective cases from January 17 to May 8, while during the same period in 2020, a total of 126 cases and 71 non-elective cases were performed. During 2020, there was a statistically significant drop in total case volume $(\mathrm{P}=0.0078)$ compared to 2019 for the time period after the declaration of COVID-19 as a national emergency (weeks 9 - 16) (Fig. 5h). Prior to the declaration of COVID-19 as a national emergency (weeks 1 - 8), there was no significant difference in weekly case volumes compared to the prior year $(\mathrm{P}=0.6)($ Fig. $5 \mathrm{~h})$. There was no significant difference in non-elective case volumes for both weeks $1-8(\mathrm{P}=1)$ and weeks $9-16(\mathrm{P}=0.375)$ between 2019 and 2020 (Fig. 6g).

\section{Plastic and reconstructive surgery}

In 2019, the plastic and reconstructive surgery service performed 179 total cases from January 17 to May 8, and 108 total cases were in 2020. The service performed 66 non-elective cases in 2019 and 62 non-elective cases in 2020. Prior to the declaration of COVID-19 as a national emergency (weeks 1
- 8), there was no significant difference in total weekly case volumes compared to the prior year $(\mathrm{P}=0.938)$ (Fig. 5i). Conversely, there was a statistically significant drop in total case volume ( $\mathrm{P}=0.0078)$ after the declaration of COVID-19 as a national emergency (weeks 9 - 16) (Fig. 5i). There was no significant difference in non-elective case volume (Fig. 6h).

\section{Surgical oncology}

A total of 140 cases were performed by the surgical oncologists from January 17 to May 8 in 2019 and 106 total cases were performed in 2020. Of those cases, 105 were non-elective in 2019 , and 74 were non-elective in 2020. Prior to the declaration of COVID-19 as a national emergency there was no significant difference in weekly total case volumes $(\mathrm{P}=0.0625)$ (Fig. $5 \mathrm{j})$, or weekly non-elective case volumes $(\mathrm{P}=0.5625)$ (Fig. 6i) compared to 2019. However, there was a statistically significant drop in both total case volumes after the declaration $(\mathrm{P}=0.0078)$ (Fig. $5 \mathrm{j})$, and in non-elective case volumes $(\mathrm{P}=0.0078)$ (Fig. 6i).

\section{Urology}

For the urology service, 181 total cases were performed between January 17 and May 8, 2019, while 172 total cases were performed in 2020 for those dates. Over that same period, the urology service performed 75 non-elective cases in 2019 and 54 non-elective cases in 2020. Prior to the declaration of COVID-19 as a national emergency (weeks $1-8$ ), there was a statistically significant increase in total weekly case volumes compared to the prior year $(\mathrm{P}=0.008)$ (Fig. 5k). There was no difference in weekly non-elective case volumes for weeks $1-8(\mathrm{P}=0.3435)$ (Fig. 6j). During 2020, there was a statistically significant drop in total case volume $(\mathrm{P}=0.0078)$ after the declaration of COVID-19 as a national emergency (weeks 9 - 16) (Fig. 5k) when compared to 2019, but not in the nonelective case volume $(\mathrm{P}=0.2188)$ (Fig. 6j).

\section{Vascular surgery}

The vascular surgery service performed 71 total cases from January 17 to May 8 in 2019 and 61 total cases in 2020. The non-elective case volumes for the period were 18 in 2019 and 19 in 2020. There was no significant difference in either total case volumes (Fig. 51) or non-elective case volumes (Fig. 6k) for this service between 2019 and 2020.

\section{Discussion}

The COVID-19 pandemic has had an unprecedented impact on healthcare delivery systems and the clinical workforce. The American surgical system adapted to the pandemic by instituting specific recommendations aimed at: 1) decreasing the transmission of COVID-19 during the preoperative, intraoperative, and postoperative period [3]; 2) limiting perioperative 
complications related to COVID-19 [4]; and 3) ensuring appropriate resource allocation and conservation during the care of surgical patients. Additionally, the ACS encouraged conservative medical management of certain diseases when appropriate in COVID-positive patients and the resumption of elective operations in a staged fashion [10]. These modifications resulted in the postponement of many elective and non-urgent surgical cases, especially at large public hospitals such as the one featured in this study. This has translated to a statistically significant decrease in both elective and non-elective case volumes. The decrease in overall volumes occurred across the majority of surgical specialties, including acute care and trauma surgery, cardiothoracic surgery, ophthalmology, oral and maxillofacial surgery, neurosurgery, otolaryngology, orthopedic surgery, plastic surgery, surgical oncology, and urology.

A governor's order canceled all elective surgeries in Texas from March 22 to April 21, 2020, so a decrease in elective cases was anticipated [11]. The city of Houston faced additional delays in resuming elective operations due to a high volume of COVID-19 cases.

It is worth noting that non-elective case volumes also had a statistically significant decline, indicating that delays in care may have occurred for more urgent surgical pathologies. This decrease was seen in the acute care/trauma surgery, surgical oncology, neurosurgery, and oral and maxillofacial surgery services. Interestingly, the orthopedic surgery service, which had seen a significant increase in non-elective cases prior to the declaration of the pandemic as a national emergency, saw a reduction in non-elective cases to levels more comparable to the prior year.

This is a critical finding as delays in care in resource-limited settings and at safety net hospitals, such as the hospital featured in this study, can result in a burdensome backlog of cases, patient loss to follow-up, and progression of pathology beyond the optimal therapeutic window for treatment. These patients frequently face significant barriers to access $[12,13]$ and disparate healthcare due to insurance status and socioeconomic factors [12, 14, 15], often manifesting in delayed presentation at baseline. Reduced case volumes secondary to the pandemic add further potential delays to care. These delays in care may have persisted, as 1 year into the pandemic the public hospital in this study continues to operate at reduced capacity due to ongoing concerns about COVID-19.

Moreover, delays in surgical care for certain pathologies, such as oncologic diseases, results in increased morbidity and mortality [7]. It has been established that patients with oncologic diseases served at safety net hospitals often present at later stages and in younger populations that are primarily racial minorities and uninsured $[13,14,16]$. Delays in care for these patients can exacerbate existent healthcare disparities and adversely affect outcomes.

It should be noted that the data for this study are limited. It analyzes surgical case volumes at one large public institution in a city with a documented high incidence of COVID-19 cases. Additionally, this data captures the initial months of the pandemic wherein little was known about the virus, its transmission, and its treatment. The statistically significant decrease in urgent cases seen between March 13 and May 8, may in part, be due to patients' decisions to postpone seeking care in order to avoid exposure in a clinical setting. It would be prudent to determine if the decrease in non-elective cases persisted for the duration of the pandemic.

Also worth mentioning is that some of the surgical subspecialties are poorly represented at this institution. For example, vascular and endocrine surgical case volumes are low at baseline resulting in the case volume analysis for these surgical subspecialties to be underpowered. Similarly, data for obstetrics and gynecology were excluded from the analysis as the service has a dedicated operating suite with separate operative case logs and restricted access. Future studies should address the impact of COVID-19 on obstetrics and gynecology case volumes as cesarean sections are the most common procedure performed globally [17]. Gynecologic cases hypothetically would have been similarly impacted potentially delaying gynecologic and urogynecologic care. It will also be particularly important to determine the health disparities that may have resulted from the postponement of surgical care during this period.

\section{Conclusions}

In facing the challenges presented by the COVID-19 pandemic, clinician leaders have been tasked with making difficult decisions regarding patient care. While leading surgical organizations provided guidelines for best practices at the start of the pandemic, the long-term implications of these decisions are unknown. This study has found that the COVID-19 pandemic has resulted in decreased performance of both elective and non-elective surgeries. This in turn raises concerns that necessary care may be delayed for marginalized populations. It is therefore incumbent on surgeons and clinicians to ensure appropriate follow-up for their patients and for healthcare systems to work towards mitigating the potential impact of case delays.

\section{Acknowledgments}

The authors would like to thank Dr. Huirong Zhu for her assistance with statistics.

\section{Financial Disclosure}

The authors have no financial disclosure for this study.

\section{Conflict of Interest}

Meeting presentation: American College of Surgeons Clinical Congress (virtual, October 23 - 27, 2021).

\section{Informed Consent}

This was an exempt, because the study is a retrospective chart review with minimal risk to patients. 


\section{Author Contributions}

Co-first author Sukriti Bansal, MD: data collection, study design, manuscript writing, editing manuscript, and data analysis. Co-first author Youmna A. Sherif, MD: IRB approval, study design, manuscript writing, editing manuscript, and data analysis. Rachel W. Davis, MD: study design, meaningful editing of manuscript. Marcia Barnett, BS, MBA: data collection and coding. Umang M. Parikh, BA: data collection and coding. Hunter Bechtold, BSA, BA: data collection and coding. Hudson Holmes, BS: data collection and coding. Yao Yang, MD, MPH: meaningful editing of manuscript, study design. David Holmes, MD: editing of manuscript. Megan $\mathrm{Vu}, \mathrm{MD}$ : editing of manuscript. Jed G. Nuchtern, MD: meaningful editing of manuscript, study design. Chad T. Wilson, MD, MPH: meaningful editing of manuscript, and study design.

\section{Data Availability}

The data supporting the findings of this study are available from the corresponding author upon reasonable request.

\section{References}

1. Hirschmann MT, Hart A, Henckel J, Sadoghi P, Seil R, Mouton C. COVID-19 coronavirus: recommended personal protective equipment for the orthopaedic and trauma surgeon. Knee Surg Sports Traumatol Arthrosc. 2020;28(6):1690-1698.

2. Bokolo AJ. Application of telemedicine and eHealth technology for clinical services in response to COVID-19 pandemic. Health and Technology. 2021;11(2):359-366.

3. Flemming S, Hankir M, Ernestus RI, Seyfried F, Germer CT, Meybohm P, Wurmb T, et al. Surgery in times of COVID-19-recommendations for hospital and patient management. Langenbecks Arch Surg. 2020;405(3):359-364.

4. Coccolini F, Perrone G, Chiarugi M, Di Marzo F, Ansaloni L, Scandroglio I, Marini P, et al. Surgery in COVID-19 patients: operational directives. World J Emerg Surg. 2020;15(1):25.

5. Collaborative CO. Mortality and pulmonary complications in patients undergoing surgery with perioperative SARS-CoV-2 infection: an international cohort study. Lancet. 2020;396(10243):27-38.

6. American College of Surgeons ASoA, Association of periOperative Registered Nurses, American Hospital Association. Joint statement: roadmap for maintaining essential surgery during COVID-19 pandemic. Published online 2020.

7. Hanna TP, King WD, Thibodeau S, Jalink M, Paulin GA, Harvey-Jones E, O'Sullivan DE, et al. Mortality due to cancer treatment delay: systematic review and meta-analysis. BMJ. 2020;371:m4087.

8. Abdallah HO, Zhao C, Kaufman E, Hatchimonji J, Swendiman RA, Kaplan LJ, Seamon M, et al. Increased firearm injury during the COVID-19 pandemic: a hidden urban burden. J Am Coll Surg. 2021;232(2):159-168. e153.

9. Ramos Perkis JP, Achurra Tirado P, Raykar N, Zinco Acosta A, Munoz Alarcon C, Puyana JC, Ottolino Lavarte P. Different crises, different patterns of trauma. the impact of a social crisis and the COVID-19 health pandemic on a high violence area. World J Surg. 2021;45(1):3-9.

10. American College of Surgeons ASoA, Association of periOperative Registered Nurses, American Hospital Association. Joint statement: roadmap for resuming elective surgery after COVID-19 pandemic. Published online 2020.

11. Abbott G. Executive Order GA-09. In: Texas OotGotSo, ed22020.

12. Gupta A, Cadwell JB, Merchant AM. Social determinants of health and outcomes of ventral hernia repair in a safety-net hospital setting. Hernia. 2021;25(2):287-293.

13. Zaidi MY, Rappaport JM, Ethun CG, Gillespie T, Hawk $\mathrm{N}$, Chawla S, Cardona K, et al. Identifying the barriers to gastric cancer care at safety-net hospitals: A novel comparison of a safety-net hospital to a neighboring quaternary referral academic center in the same healthcare system. J Surg Oncol. 2019;119(1):64-70.

14. Bradley CJ, Dahman B, Shickle LM, Lee W. Surgery wait times and specialty services for insured and uninsured breast cancer patients: does hospital safety net status matter? Health Serv Res. 2012;47(2):677-697.

15. Farooq A, Paredes AZ, Merath K, Hyer JM, Mehta R, Sahara K, Tsilimigras DI, et al. How safe are safety-net hospitals? Opportunities to improve outcomes for vulnerable patients undergoing hepatopancreaticobiliary surgery. J Gastrointest Surg. 2020;24(11):2570-2578.

16. Lee RM, Gamboa AC, Turgeon MK, Yopp A, Ryon EL, Kronenfeld JP, Goel N, et al. Dissecting disease, race, ethnicity, and socioeconomic factors for hepatocellular carcinoma: An analysis from the United States Safety Net Collaborative. Surg Oncol. 2020;35:120-125.

17. Lin Y, Dahm JS, Kushner AL, Lawrence JP, Trelles M, Dominguez LB, Kuwayama DP. Are American surgical residents prepared for humanitarian deployment?: A comparative analysis of resident and humanitarian case logs. World J Surg. 2018;42(1):32-39. 